\title{
Orientation and Control of a Flexible Spacecraft : Planar Motion
}

\author{
Ömer Morgül \\ Department of Electrical and Electronics Engineering \\ Bilkent University \\ PK 8 Maltepe, 06572, Ankara, Turkey
}

Abstract

We consider a flexible spacecraft modeled as a rigid body which rotates in an inertial frame; a light flexible beam is clamped to the rigid body at one end and free at the other end. We assume that the flexible spacecraft performs only planar motions. We pose two control problems; namely, the orientation and the stabilization of the system. It is shown that suitable boundary controls applied to the free end of the beam and suitable control torques applied to the rigid body solve the problems posed above.

\section{Introduction}

Many mechanical systems, such as spacecraft with flexible appendages or robot arms with flexible links, can be modeled as coupled elastic and rigid parts. Many future space applications, such as the space station, rely on lightweight materials and high performance control systems for high precision pointing, tracking, etc. , and to achieve high precision demands for such systems, one has to take the dynamic effect of the flexible parts into account. Thus, over the last decade there has been a growing interest in obtaining new methods for the design, analysis and control of the systems which has flexible parts.

In this paper, we model a flexible spacecraft as a rigid body with a flexible beam clamped to it at one end, the other end of the beam is free, and study the motion of this system. We pose two control problems : the orientation and the stabilization of the system. To solve these problems, we propose two types of control laws : one based on cancellation and one we call natural. In each case, we propose a boundary force and a boundary torque control, both applied at the free end of the beam, and a torque control applied to the rigid body.

In section 2, the equations of motion are given. In section 3 and 4 , the proposed control laws are given and main results are presented.

\section{Equations of Motion}



Figure 1: Rigid body with a flexible beam

\footnotetext{
${ }^{\circ}$ This research has been partially supported by the National Science Foundation Grant ECS 8500993 and by the Scientific and Technical Research Council of Turkey.
}

We consider the configuration shown in the figure 1 :

In figure $1,\left(O, \mathbf{e}_{1}, \mathbf{e}_{2}, \mathbf{e}_{3}\right)$ denotes a dextral orthonormal inertial frame, which will be referred to as $\mathbf{N},\left(O, \mathbf{D}_{1}, \mathbf{D}_{2}, \mathbf{D}_{3}\right)$ denotes a dextral orthonormal frame fixed in the rigid body, which will be referred to as $B$, where $O$ is also the center of mass of the rigid body and the axes $D_{1}, D_{2}, D_{3}$ are along the principal axes of inertia of the rigid body. The beam is clamped to the rigid body at the point $Q$ at one end along the $\mathbf{D}_{2}$ axis and free at the other end. $\theta$ is the angle of rotation of the rigid body. We assume that the rigid body may rotate about the $\mathbf{e}_{1}$ axis only and that at all times the axes $\mathbf{e}_{1}$ and $\mathbf{D}_{1}$ coincide. We also assume that the mass of the rigid body is much larger than the mass of the beam, hence the center of mass of the rigid body is approximately the center of mass of the whole system.

Let $L$ be the length of the beam. We assume that the beam is inextensible, (i.e. no deformation along the axis $\mathbf{D}_{2}$ ), and homogeneous with uniform cross-sections. The beam is initially straight along the $D_{2}$ axis. This initial configuration of the beam is also referred to as the reference configuration. Let $P$ be a beam element whose distance from the point $Q$ in the reference configuration is $x$, let $u$ be the displacement of $P$ along the $\mathrm{D}_{3}$ axis.

Neglecting gravitation, surface loads and the rotatory inertia of the beam cross-sections, the relevant equations of motion of the system are : for $x \in(0, L), t \geq 0,[2],[3]$

$$
\begin{array}{cc}
\rho u_{t t}+E I u_{x x x x}+\rho \ddot{\theta}(b+x)-\rho \dot{\theta}^{2} u=0, \quad 0<x<L \\
I_{R} \ddot{\theta}=E I\left(-b u_{x x x}(0, t)+u_{x x}(0, t)\right)+N_{c}(t) \\
u(0, t)=0, \quad u_{x}(0, t)=0 \\
-E I u_{x x x}(L, t)=f_{1}(t) \quad, \quad E I u_{x x}(L, t)=f_{2}(t)
\end{array}
$$

where $\rho$ and $E I$ are the mass per unit length and the flexural rigidity of the beam, respectively, both assumed to be constant, $N_{c}(t)$ is the torque applied to the rigid body, $f_{1}(t)$ and $f_{2}(t)$ are the boundary control force and torque, respectively, both applied at the free end of the beam.

We now pose the following two control problems on the system given by (1)-(4):

Problem 1: (orientation problem) Consider the system given by (1)-(4). Let an angle $\theta_{0} \in[0,2 \pi)$ be given. Find appropriate control laws for $N_{c}(t), f_{1}(t)$ and $f_{2}$ such that the solutions $u(x, t), u_{t}(x, t)$ and $\theta(t)$ of $(1)-(4)$ satisfy the following asymptotic relations :

$$
\begin{array}{lll}
\lim _{t \rightarrow \infty} u(x, t)=0 & , & 0 \leq x \leq L \\
\lim _{t \rightarrow \infty} u_{t}(x, t)=0 & , & 0 \leq x \leq L \\
\lim _{t \rightarrow \infty} \theta(t)=\theta_{0} & & \\
\lim _{t \rightarrow \infty} \dot{\theta}(t)=0 & &
\end{array}
$$

where the angle $\theta_{0}$ is the angle along which we want to align the $\mathrm{D}_{2}$ axis. $\square$

Problem 2 : (stabilization problem) Consider the system given by (1)-(4). Find appropriate control laws for $N_{c}(t), f_{1}(t)$ and $f_{2}$ such that the solutions $u(x, t), u_{t}(x, t)$ and $\theta(t)$ of (1)-(4) satisfy the following asymptotic relations :

$$
\begin{array}{lll}
\lim _{t \rightarrow \infty} u(x, t)=0 & , & 0 \leq x \leq L \\
\lim _{t \rightarrow \infty} u_{t}(x, t)=0 & , & 0 \leq x \leq L \\
\lim _{t \rightarrow \infty} \dot{\theta}(t)=0 & &
\end{array}
$$

口 
In the following sections, we propose two types of control laws, one we call natural and one based on cancellation to solve the problems posed above. In both of these control laws, we choose $f_{1}(t)$ and $f_{2}(t)$ as follows :

$$
f_{1}(t)=-\alpha u_{t}(L, t) \quad, \quad f_{2}(t)=-\beta u_{x t}(L, t)
$$

where $\alpha$ and $\beta$ are arbitrary positive constants.

\section{Natural Control Laws}

Control Law 1: (for orientation problem) Together with the boundary control (5), we propose the following control torque for $N_{c}(t)$ :

$$
\begin{gathered}
N_{c}(t)=E I\left((b+L) u_{x x x}(L, t)-u_{x x}(L, t)\right) \\
-k_{1} \dot{\theta}(t)-k_{2}\left(\theta(t)-\theta_{0}\right)
\end{gathered}
$$

where $k_{1}$ and $k_{2}$ are arbitrary positive real constants. We call the control law given by (5)-(6) natural control law because it enables one to use the energy of the whole configuration as a Lyapunov function to study the stability of the system.

The "energy" norm associated with the system given by $(1)-(6)$ is

$$
\begin{gathered}
E:=\frac{1}{2} I_{R} \dot{\theta}_{e}^{2}+\frac{1}{2} \rho \int_{0}^{L}\left[\dot{\theta_{e}^{2}} u^{2}+\left(u_{t}+(b+x) \dot{\theta_{e}}\right)^{2}\right] d x \\
+\frac{1}{2} E I \int_{0}^{L} u_{x x}^{2} d x+\frac{1}{2} k_{2} \theta_{e}^{2}
\end{gathered}
$$

where $\theta_{e}:=\theta(t)-\theta_{0}$. The first term in (7) represents the rotational kinetic energy of the rigid body, the second term represents the kinetic energy of the beam in the inertial frame $\mathrm{N}$, the third term represents the potential energy of the beam and the last term is a measure of "orientation energy", in the sense that when $k_{2} \theta^{2}=0$ on $\mathbf{R}_{+}$, then the correct orientation is achieved, i.e. $\theta(t)=\theta_{0}$, $t \geq 0$.

By differentiating (7) with respect to $t$, and using (1)-(6), we obtain :

$$
\dot{E}=-k_{1} \dot{\theta}_{e}^{2}-\alpha u_{i}^{2}(L, t)-\beta u_{x t}^{2}(L, t)
$$

which shows that the system given by (1)-(6) is dissipative, [2] Actually we have:

Theorem 1 : The energy $E$ given by (7) asymptotically decays to zero along the solutions of (1)-(6).

Proof : The proof of this theorem follows from (8) and from LaSalle's invariance theorem generalized to infinite dimensional spaces, [5]. For details, see [2].

Control Law 2 : (for stabilization problem) If we are interested only in stabilization, but not in orientation, we can modify $(1)-(6)$ by setting $k_{2}=0$. More precisely, together with (5), we propose the following control law for $N_{\mathrm{c}}(t)$ :

$$
N_{c}(t)=E I\left((b+L) u_{x x x}(L, t)-u_{x x}(L, t)\right)-k_{1} \dot{\theta}(t)
$$

where $k_{1}$ is an arbitrary positive constant. In this case, the result of theorem 1 is still valid. Moreover, we have :

Theorem 2 : Consider the energy $E(t)$ given by $(7)$, with $k_{2}=0$. Then, the energy decays exponentially to zero along the solutions of (1)-(5), (9).

proof : for details, see [3]. $\square$

\section{Control Laws Based on Cancellation}

Control Law 3: (for orientation) Together with the boundary control (5), we propose the following conrol law for $N_{c}(t)$ :

$$
N_{c}(t)=E I\left(b u_{x x x}(0, t)-u_{x x}(0, t)\right)-k_{1} \dot{\theta}(t)-k_{2}\left(\theta(t)-\theta_{0}\right)(10)
$$

where $k_{1}$ and $k_{2}$ are arbitrary positive constants.

Note that, when (10) is substituted in (2), the torque effect of the beam on the rigid body (i.e. the first two terms in the right hand side of (2)) will be cancelled by the first two terms in the right hand side of (10); hence the name cancellation. This results in a rigid body equation decoupled from the beam, which yields solutions $\theta_{e}(t)$ decaying exponentially to zero. When this known solution $\theta_{e}(t)$ is substituted in (1), by using a result from [1], it can be shown that the energy :

$$
\tilde{E}=\frac{1}{2} \rho \int_{0}^{L} u_{t}^{2} d x+\frac{1}{2} E I \int_{0}^{L} u_{x x}^{2} d x
$$

associated with the beam deflections also decays exponentially to zero. For details, see [3], [4].

Control Law 4: (for stabitization) As in control law 2, by setting $k_{2}=0$ in $(10)$, one can achieve stabilization. More precisely, together with (5), we propose the following control law for $N_{c}(t)$ :

$$
N_{c}(t)=E I\left(b u_{x x x}(0, t)-u_{x x}(0, t)\right)-k_{1} \dot{\theta}(t)
$$

where $k_{1}$ is an arbitrary positive constant. It can be shown that, $\dot{\theta}_{e}(t)$ and the energy $\tilde{E}$ given by (11) decays exponentially to zero. along the solutions of (1)-(5), (12). For details, see [3], [4]. $\square$ remark 1 : In all the control laws proposed here, if one chooses $f_{2}=0$, or equivalently $\beta=0$, the conclusions of theorems 1 and 2 and the results corresponding to the cancellation scheme still hold, [3], [4].

\section{Conclusion}

In this paper, we studied the planar motion of a flexible beam clamped to a rigid body. We assumed that the center of mass of the rigid body is fixed in an inertial frame, and that the configuration performs only planar motions. We posed an orientation and a stabilization problem, and to solve this problems we proposed four control laws. In the case of control law 1, the orientation problem is solved with an asymptotic decay of the energy, (see Theorem 1), whereas in the remaining control laws this decay is exponential. Application of similar control laws proposed in this paper for a flexible spacecraft without assuming that the center of mass of the rigid body is fixed in an inertial frame will be the subject of another paper. Finally, as a future research, we conjecture that, the asymptotic stability result stated in the Theorem 1 can be extended to an exponential stability result without any change in the hypothesis.

\section{References}

[1] G. Chen et. al. , "Modelling, Stabilization and Control of Serially Connected Beams," SIAM J. of Contr. and Opt., vol.25, pp.526-546, 1987.

[2] Ö. Morgül, "Orientation and Stabilization of a Flexible Beam Attached to a Rigid Body : Planar Motion," submitted to IEEE $T-A C$.

[3] Ö. Morgül, "Control and Stabilization of a Flexible Beam Attached to a Rigid Body," Int. J. of Cont., vol. 51, No. 1, pp. 11-31, 1990 .

[4] Ö. Morgül, Problems in the Control of Flexible Spacecraft, Ph.D. Thesis, Memorandum No. UCB/ERL M89/97, University of California, Berkeley, 1989.

[5] S. Saperstone, Semidynamical Systems in Infinite Dimensional Spaces, New York, Springer-Verlag, 1981 